\title{
Application of Invigorating Qi and Moistening Intestines Method in Qi Deficiency Constipation
}

\author{
Wenxin $\mathrm{Li}^{1 \text {,** }}$, Hua Jiang ${ }^{2}$, Zongrun $\mathrm{Li}^{1}$, Liangwu Huang ${ }^{1}$ \\ ${ }^{1}$ Shaanxi University of Chinese Medicine, Xianyang 712000, Shaanxi, China. \\ ${ }^{2}$ The Affiliated Hospital of Shaanxi University of Chinese Medicine, Xianyang 712000, Shaanxi, China.
}

\begin{abstract}
How to cite this paper: Wenxin $\mathrm{Li}$, Hua Jiang, Zongrun Li, Liangwu Huang. (2022) Application of Invigorating Qi and Moistening Intestines Method in Qi Deficiency Constipation. International Journal of Clinical and Experimental Medicine Research, 6(1), 60-67. DOI: 10.26855/ijcemr.2022.01.011
\end{abstract}

Received: November 22, 2021

Accepted: December 17, 2021

Published: January 5, 2022

*Corresponding author: Wenxin $\mathrm{Li}$, Shaanxi University of Chinese Medicine, Xianyang 712000, Shaanxi, China. Email: 1632625581@qq.com

\begin{abstract}
Based on the understanding of Traditional Chinese medicine and Western medicine on the etiology and pathogenesis of functional constipation and the treatment methods for internal and external use. Based on the theory that "more than cure all diseases is born from qi", the formation of qi deficiency constipation and its important position in constipation are discussed. Fully understand the role of spleen and stomach in constipation, get through invigorating the spleen and stomach on the basis of qi to achieve the function of the fu stagnation, moistening the bowel bowel laxation. BuzhongYiqi Decoction is a representative prescription of invigorating qi and raising Yang and warming and removing heat. It is analyzed as a classic prescription of qi deficiency and secret, to explore the clinical application and clinical efficacy of qi replenishing formulas such as Huangqi Decoction. To summarize the application of nourishing qi and moistening intestines in the treatment of qi-deficiency constipation.
\end{abstract}

\section{Keywords}

Invigorating qi and moistening intestine method, Replenishing qi prescription for qi deficiency constipation, clinical application

Constipation is a symptom caused by the pathological process of a variety of diseases [1], manifested as difficult fecal discharge, dry and hard stool, reduced defecation times, increased defecation time, anus blockage during defecation, incomplete defecation, etc., sometimes accompanied by abdominal pain and abdominal distension. Clinically, constipation can be classified into functional constipation and organic constipation according to the presence or absence of organic diseases. According to the Rome iii criteria in Internal Medicine [2]: symptoms began to appear 6 months ago, and $\geq 2$ of the following symptoms were met in the last 3 months: 1 . 2, at least $25 \%$ of the defecation is dry ball stool or hard stool; 3 . At least $25 \%$ of bowel movements have a sense of inadequacy; 4. At least $25 \%$ of bowel movements have a sense of anal rectum obstruction or obstruction; 5 . At least $25 \%$ of defecation needs manual help; 6 . Defecation $<3$ times per week; 7 . Loose stools rarely occur without laxatives and do not meet the diagnostic criteria for irritable bowel syndrome (IBS). Along with the social development, people living standard rise, the common thing of meat products is on the table, there are some fast food, and people in the process of pursue pyramid end life affected by mental and social factors, the prevalence of constipation in recent years has been on the rise, and prevalence increases with the increase of age [3]. Generally speaking, the prevalence rate in European and American countries is higher than that in Asian countries [4], which is due to dietary differences. Westerners prefer fried and fast food, which will bring overload pressure to the intestine. Female prevalence than men, with the, belt, tyres, and other special physical production, women than men are more prone to blood deficiency symptoms, and intestinal lose moist embellish cause constipation, women have the characteris- 
tics of "yu", in the face of various pressure, relative to men, are more likely to appear nervous depression, liver advocate of modern, diseases with stasis, e. jet fluid, Causes constipation [5]. What is more, liver yu day is long heat, burning body fluid, dry large intestine, no water boat will lead to constipation. The prevalence rate of middle-aged and elderly is higher than that of young people. Compared with young people, middle-aged and elderly people are weak in constitution, lack of qi, small amount of exercise, weak intestinal creep force and prone to constipation.

\section{Understanding of constipation in modern medicine}

\subsection{The cause}

At present, the pathogenesis of functional constipation is not clear, which may be the result of the interaction of many factors. The causes of neurological, endocrine and metabolic abnormalities are as follows:

\subsubsection{Diet and alcohol consumption}

Poor diet, changes in diet structure and low water intake will increase the possibility of constipation. Abstemious eating includes dieting and overeating. Changes in diet structure include a preference for spicy, low-calorie, mono-diet, and a preference for refined diet. With the development of economy, people no longer eat for full stomach, some people choose to enjoy delicious food to their heart's content, without any dietary restrictions, sweet and spicy; some people choose to be vegetarian for the sake of health and rarely touch meat. Also some people pay attention to own body appearance, listen to some weight loss experts on the network, take the "apple law reducing weight”, "August 16th law reducing weight", "light is broken feed” and other methods and excessive dieting, or only eat a low calorie diet control calories and achieve the goal that reduce weight, was damaged in the process of their health. Wine is also spicy things, regular drinking will not only cause damage to the gastric mucosa, resulting in gastritis, stomach ulcers, but also cause additional burden on the liver, leading to alcoholic liver, cirrhosis, but also cause constipation. Intake of food too little, after enter the stomach digestion, into the intestinal scraps of food too little, or too little food intake and dietary fiber food partial heavy, lead to intestinal chyme and fecal mass quantity is not enough to stimulate the intestinal peristalsis causes meaning is normal and defecation reflex, intestinal load capacity is reduced, the stimulus of the gut is weak, that cause constipation. The amount of drinking water is too little, the lack of moisture in the intestine, the moisture in the stool is also absorbed by the intestine, the water content is reduced, and it becomes dry and hard, resulting in constipation.

\subsubsection{Psychological factors}

Contemporary society there is competition everywhere, everyone there are different pressure, the pressure on students with learning, workers have more work, and family economic pressure, so the lack of sleep, long-term is in nervous condition, mental depression, anxiety, resulting in a decline in central adjustment ability, affect the endocrine and the function of autonomic nervous system, causing intestinal function change, constipation occurs. According to the survey of college students in Wuhan [6], the prevalence of constipation among college students is higher than that of the general population, while the prevalence of constipation among undergraduates, master's students and doctoral students is gradually decreasing, which may be due to the fact that the study pressure and employment pressure of undergraduates are significantly higher than those of master's and doctoral students.

\subsubsection{Bowel habits}

Having good bowel habits is one way to avoid constipation. The advantage of scheduled defecation is that the internal biological clock system senses the defecation time and stimulates the intestinal contraction on time, promoting intestinal peristalsis. Colon movement is most active after getting up and eating in the morning. Drink a cup of plain water to promote intestinal peristalsis. A survey of the non-patient population in Beijing [7] showed that up to $77 \%$ of the population defecated in the morning and morning, and $84 \%$ of the population could guarantee to defecate once a day.

\subsubsection{Environmental factors}

Constipation is also linked to environmental factors. Cui Yuhong [8] conducted relevant research on constipation of 247 female students in her school. The study found that the number of female students who just entered the school due to environmental changes, constipation accounted for $64.4 \%$, it is concluded that environmental change is one of the most common factors of constipation. Yu Pulin et al. [9] found in their study that the incidence of constipation in north China was higher than that in south China, and that in rural areas was higher than 
that in cities.

\subsubsection{Drugs}

Clinically, there are many side effects of drugs that will cause constipation, among which the most common is long-term use of opioid drugs for pain relief. Opioid drugs combine with intestinal opioid receptors to slow intestinal peristalsis, delay fecal excretion time, and reabsorb the water in feces in the intestinal tract. Finally, feces become dry and hard, making excretion difficult [10]. Or some have constipation of patients, without the system of treatment, choice of long-term colonics or using laxatives, such meetings produce drug dependence, reflect rectal sensitivity is abate, seemingly daily defecate is normal, but the symptoms of constipation in growing, once stop drug, will find that is more serious than medication before symptoms, moreover may not completely independent, defecate cannot get away from using laxatives.

\subsection{Treatment}

There is no special treatment for constipation, the main treatment is patient education, diet and lifestyle adjustment, such as paying attention to the amount of diet, enough volume can form dregs in the intestinal tract to stimulate intestinal peristalsis; pay attention to diet quality, eat more coarse grains and dietary fiber food; keep enough water, develop the habit of regular defecation, do not artificially restrain the intention of defecation, do not read or read newspapers when defecating, adjust the lifestyle, go to bed early and get up early, maintain optimistic mental state, increase outdoor sports, etc. If serious, can be medicine, at present mainly laxative, divided into stimulating a laxative, volumetric cathartic, lubricity laxative, stimulating a laxative with phenolphthalein, double vinegar benzene organism, lubricity laxative lactulose, magnesium sulfate, polyethylene glycol, lubricity, a laxative, such as open plug, also can use gastrointestinal medicine such as mo sand Billy, xisha Billy, etc. But western medicine treatment is prone to some uncertain adverse reactions. For example, abuse of laxatives can cause lasting damage to neurons in the colon wall, repeated symptoms and drug dependence after withdrawal. And traditional Chinese medicine thinks constipation, caused by a deficiency of qi and blood, Yin and Yang, the obvious advantages compared with western medicine on treatment of dependence is small, stable curative effect, prognosis is good, can according to the different symptoms of patients showed the treatment based on syndrome differentiation, argues that "this" pay attention to the specimen, cure, improve their lung, spleen and kidney function of relieving constipation in patients with the improved.

\section{TCM's understanding of constipation}

\subsection{Tracing of disease name}

Constipation [11] was first recorded in Huangdi Neijing, which is called "post-adverse". In treatise on Febrile diseases, Zhang Zhongjing referred to constipation as Yin knot, Yang knot, spleen covenant and closure. In the Tang Dynasty, Sun Simiao divided constipation into "difficult defecation" and "impassability of defecation" according to the difference between the severity of the disease in his "Prescriptions for Urgent and Precious Gold". "Danxi Heart method" will be called constipation "dry stool". All the words in the ancient books above describe the symptoms of constipation. As for the word "constipation”, it was first mentioned in the Origin of Miscellaneous Diseases in the Qing Dynasty, which was recognized by later generations of doctors and thus used today, and the definition of "constipation" was improved.

\subsection{Understanding of etiology, pathogenesis and clinical manifestations [12]}

Although constipation is located in the large intestine, it is closely related to the functions of lung, spleen, stomach, liver and kidney as well as qi, blood, body fluid and emotion.

\subsubsection{Damp heat bet}

Always have a taste for spicy and hot products, spicy things are easy to brew wet heat, damp evil heavy stagnation, heat evil burning body fluid, consumption of gas injury, intestinal dryness and demoistening. The expression is defecate difficulty, there is obstruction inside rectum, drop to rise a feeling, perineum burning sense excrement clamps have mucous, mouth is dry, tongue is red, moss is yellow greasy.

\subsubsection{Stagnant air}

Study or most work need to sit for a long time, plus all kinds of pressure in life, work and family caused by ex- 
cessive worry and worry, mood is not free, the spirit to maintain a high state of tension, repression of qi, leading to qi stagnation, cannot be announced, stasis in the body, intestinal damage, body imbalance, waste excretion is not free. It is manifested as normal emotional abnormalities, such as irritability and sadness, frequent belching, constipation, difficult excretion, fullness of chest and hypochasm, abdominal distension, bitter mouth, weak tongue, and string of pulse.

\subsubsection{Yang deficiency cold coagulation}

Yang deficiency weak, work too much or high body failure, long illness, will dissipate Yang qi, Yin cold endogenous, stay in the gastrointestinal, aggravating Yang qi injury, stagnation, body fluid is not good, conduction disorders. Clinical manifestations are as follows: difficult stools, difficult discharge, long and clear urination, pale complexion, fear of cold and warm, accompanied by cold abdominal pain [13].

\subsubsection{Spleen deficiency and qi depression}

Old and weak, qi and blood recession, or a woman childbirth consumption injury of a large number of qi and blood, positive qi has not been recovered, or gastrointestinal diseases, spleen and stomach weakness, can lead to insufficient gas, lift weakness, solid loss of department, large intestine conduction weakness, constipation. Performance is not dry stool, the note is not thick, or dry, such as chestnut, difficult discharge, defecation is unable to earn, earn sweat shortness of breath, after the stool is weak, accompanied by god fatiguing qi, eat less to stay.

\subsubsection{Deficiency of qi and Yin}

Year high body empty, after illness, postpartum, or internal injuries, weary diet lead to qi and Yin, qi jin, thirst, the deficiency of body fluid deficiency, kidney Yin deficiency can lead to lack of body fluid, intestinal fluid embellish speech \& drama, lost the final performance for: less defecation, stool stem node, defecation, shortness of breath, move the sweat out, dry mouth, tongue thin body, less moss, pulse fine.

\section{TCM treatment}

\subsection{Traditional Chinese medicine for internal and external use}

\subsubsection{Internal and}

LvRuimin [14] used flavoring ShengyangYiwei Decoction (Shuimi pills): Astragalus membranaceus, Sundried ginseng, Herbaceous peony, Tangerine peel, Poriacocos, Bupleurum bupleurum, Immaturusaurantii, Alisma alismatifolia, RhizomaMultiflorum, Atractylodesatractylodes, licorice, trichosanthin, Panax notoginseng, Deserticolacistanche, cynomoriumcynomorium. Control group oral lactulose, treatment group effective rate of $90 \%$, much higher than the control group. Chen Xuan et al. [15] used Maziren pill combined with lactudrop, while the control group only used lactudrop. The effective rate of the treatment group was $96.61 \%$, higher than that of the control group (84.75\%) by comparing the colon transport time and serum intestinal neurotransmitter indexes before and after medication.

\subsubsection{Attach}

Ning Yuyin et al. [16] used WenyangYiqi ointment applied to umbilical ring as the observation group, and Rhubarb ointment applied to Shenque, a classic prescription for treating constipation, as the control group. Both groups of plaster were effective, and wenyangYiqi ointment applied to umbilical ring was superior to the control group in increasing defecation times and improving constipation symptoms. Wang Haifang et al. [17] applied new acupuncture and moxibustion sticking tianshu point combined with vinegar treating Rhubarb sticking Shenque point to treat functional constipation in the elderly, with an effective rate of $96.67 \%$.

\subsection{Acupuncture and massage treatment}

Huang Cuichang et al. [18] applied ear point pressing bean: large intestine, rectum and sanjiao as main points, lung, spleen, kidney and stomach as matching points, combined with hand acupoints: Shangyang point, Big intestine point, small intestine point, Erjian point, Sanjian point, sanjiao point and colon area massage to treat qi deficiency and constipation in the elderly, with a total of 60 cases and an effective rate of 93.4\%. Liu Li et al. [19] used acupuncture to select acupoints: Tianshu, Xiawan, Qihai, Guanyuan, Zhigou, Yanglingquan and other acupoints. Combined with conventional treatment, the effective rate was 90.77\%. Lin Linyan and Liu Kunming [20] chose the mountain fire acupuncture method as the observation group and the conventional acupuncture method as the control group. Points were selected in both groups: Tianshu, Danshu, Zusanli, Shangjuxu and Zhigou. The 
effective rate was 93.33\%, much higher than that of control group.

\section{Treatment of qi deficiency syndrome}

The etiology and pathogenesis of constipation have different views of doctors in past dynasties, and their treatment varies from person to person. Constipation is generally divided into positive and deficiency syndrome. Deficiency syndrome, mostly due to weak body, external sensory six erotic, diet fatigue internal injury or postpartum, disease, and old body deficiency, deficiency of Yin and Yang qi and blood, resulting in impotence of stool. Yang Qi deficiency results in warm and weak transmission, while Yin blood deficiency results in intestinal loss [21]. "Symptom due to pulse treatment constipation conclusion" in the description of "if the vitality is insufficient, lung qi cannot be issued, the large intestine cannot preach the order, and stool also knot. It shows that deficiency of qi qi affects various viscera meridians, thus deficiency of Qi and blood Yin and Yang affects physiological function of large intestine. Treatment should be treated to seek this, specimens treated, dispel evil and help healthy qi, according to the different symptoms of the diagnosis of qi and blood Yin and Yang deficiency of one or more, using nourishing Yin and blood, yiyang temperature method. The clinical manifestations of qi deficiency syndrome in deficiency syndrome are mainly dry stool, rough notes, difficult excretion, sweat and exertion, shortness of breath and weakness, pale complexion, fatigue, lack of qi and lazy speech, weak pulse, pale tongue, nourishing qi and moistening intestine [22].

\subsection{The basis of nourishing spleen and stomach qi and runchang method}

Qi is the basic substance of the human body. "Su Ask Lift pain” cloud: "More than cure all diseases born in qi." It indicates that the disorder of qi is the basic pathogenesis of disease, and it leads to a variety of diseases, constipation is no exception [23]. Constipation has a long and recurrent course, mostly caused by deficiency of Qi, blood and Yin and Yang of zang-fu organs. The occurrence of the disease and lung, spleen, stomach, liver, and kidney fu dysfunction, especially closely related to the spleen and stomach. Because the spleen and stomach belong to the middle soil, the middle soil is the axis of the four transport, which is delivered to the lung in the upper part, benefiting the liver and kidney in the lower part, nourishing the four sides of the external irrigation, nourishing the yingwei and nourishing the stomach in the spleen, so that the grain qi is strong, qi and blood are sufficient, and all the five zang organs are safe [24]. The spleen is the main source of cleansing, transportation, rising temper, and upward transmission of water and grain fine substances to other zang-fu organs to nourish the whole body, which is the origin of acquired life and the biochemical source of qi and blood [25]. The stomach is the main receiver of decomposed food, like moistening and evil dryness, and the stomach qi is the main drop. Together with the spleen, it is the hub of qi movement, which can lift up qingyang, and go down turbidity. If the spleen and stomach are hurt, the qi is insufficient, the vitality cannot be generated, the function of the viscera is damaged, and all diseases are born. Treatment of constipation, can start from the spleen and stomach, tonifying the spleen and stomach of qi, qi machine operation, blood, viscera function to return to normal. The method of nourishing qi and moistening intestines is represented by buzhong Nourishing Qi Decoction.

\section{Prescription analysis and clinical application of BuzhongYiqi Decoction}

\subsection{Recipe Analysis}

BuzhongYiqi Soup is selected from The Theory of Discrimination and Confusion of Internal and External Injuries, which is founded on li Dongyuan's spleen and stomach theory and deeply contains the theory of the rise and fall of Yin and Yang [26]. For qi-deficiency constipation, BuzhongYiqi Decoction is treated with "tonic", "xing" and "embellishment" [27]. Astragalus in the prescription for king medicine, can fill a body of gas, Yang, real wei qi, solid surface antiperspirant, but also can produce blood. With ginseng, prepared glycyrrhiza, reuse three drugs, the three are "sweet and warm Yang medicine also", take its sweet and warm spleen and stomach and angry; With atractylodesatractylodes, invigorating the spleen dryness wet li shui, but also can and stomach shengjin, for tonifying temper to medicine. Sheng Baizhu qi tonifying the spleen, moistening the intestine to relieve constipation, at the same time to protect the spleen and stomach, is "the essential medicine of qi deficiency constipation". Tangerine peel for qi medicine, spleen and stomach qi, can strengthen the spleen. The orderly rise and fall of Radix bupleurum and Radix flax have the effect of raising the Yang and lifting the depression, and can lead the clear qi of lifting and sinking to the spleen and stomach, and can raise the sweet and warm qi of Astragalus membranaceus, ginseng and licorice, and supplement the qi of lung and wei to strengthen the surface [28]. Because blood is the mother of qi, blood can nourish qi, blood can carry qi. As constipation lasts for a long time [29], jin qi is depleted, 
and jin blood cannot nourish the large intestine. Therefore, Angelica is used to nourish blood and promote blood circulation, nourish blood, moisten intestine and relieve constipation, and nourish qi by nourishing blood. Combined with ginseng and Astragalus, angelica can enhance the effect of replenishing qi and also moisten intestine and relieve constipation. In buzhongYiqi decoction, supplementing qi medicine with a small amount of hemp, Bupleurum, buzhongYisheng, enhance the function of the prescription of raising Yang lift depression. At the same time supplementing gas drugs with a small amount of gas drugs, supplementing without stagnation. The combination of all drugs to raise clear and reduce turbidity, qi and blood, so that the blood is full, qi and blood enough, the intestinal tract moist, zang-fu qi machine up and down orderly.

\subsection{Clinical application of BuzhongYiqi Decoction:}

(1) Gong Liyan [30] treated 72 elderly patients with habitual constipation, including 30 cases of qi deficiency, 19 cases of qi and blood deficiency and 23 cases of Yang deficiency. Two groups were randomly divided into two groups, the treatment group and the control group were treated with mosabili tablet, the treatment group was added with BuzhongYiqi decoction, and the patients with qi deficiency were added with ZHishi and Maren. With deficiency of qi and blood and fructus aurantii, flaxseed, peach kernel, fructus aurantii; Both Yang deficiency and cistanche, cooked aconite, cinnamon. The results showed that the total effective rate of the treatment group was up to $97.22 \%$. BuzhongYiqi decoction has a good effect on the treatment of old habitual constipation. (2) Liang Peiling et al. [31] believed that chronic functional constipation is mostly seen in patients with old age or chronic physical deficiency, or in women who consume qi and hurt blood after childbirth, resulting in deficiency of qi and blood, deficiency of Yin fluid, and in severe cases, fire of Yin deficiency, resulting in intestinal dryness and difficulty in defecation, forming the syndrome of qi deficiency and intestinal dryness mixed with deficiency and reality. They selected 100 patients with chronic functional constipation and randomly divided them into two groups. The treatment group and the control group were treated with phenolphthalein tablets, and the treatment group was treated with BuzhongYiqi Decoction and Maziren pills. For the constipation patients with severe qi Yin deficiency and vigorous empty fire, they should first reduce their empty fire and moisten dryness, and then fill up their gas. First use pockseed pills to increase Yin and liquid, moisten dryness and relieve heat, and then use buzhongYiqi tang Yiqi moisten intestines and relieve constipation, both qi and Yin take into account.

\section{The clinical application of other formulas for supplementing qi and moistening intestine}

\subsection{Replenishing qi and moistening intestines}

Song Liying et al. [32] summarized 60 cases of qi deficiency constipation treated with their self-designed YiqiRunchang prescription. The basic ingredients of the formula for nourishing qi and moistening intestine are Astragalus membranaceus, tangerine peel, hemp seed, Angelica root, peony root, Dangshen, Cistanchedeserticola, black sesame, Atractylodesmacrocephala and almond. Add fried malt and amomum for less abdominal distension; Abdominal distension, white greasy tongue with white lentil, coix seed; Restless plus cypress seed, jujube seed; Fatigue sweat plus windbreak. The results show that ideal results are obtained.

\subsection{Replenishing qi and moistening intestine tongfu Decoction}

Hong Yanghua et al. [33] believed that weakness of spleen and stomach and weak conduction of large intestine were the main reasons for immobility of stool and retention in intestine. They treated and observed 60 cases of colon slow transport type qi-deficiency constipation. The control group was treated with Huangqi Decoction, a representative prescription of Qi deficiency and mi, and the treatment group was treated with YiqiRunchangTongfu Decoction. The basic ingredients of qi nourishing and chang Nourishing decoction are Astragalus membranaceus, Codonopsispilosula, Huang Jing, Atractylodesatractylodes, Radix Ophiopogonis, Cistanchedeserticola, Angelica sinensis, Hemp seed, AurantiiAurantii and Magnolia officinalis. Prescription reuse of qi tonic medicine, qi tonic stronger than astragalus decoction, promote large intestine conduction, but also enhance the immune function of the human body. Radix ophiopogonis, Shengdi and Yuanshen are added to the astragalus decoction to increase intestinal moisture and promote defecation. The results showed that the total effective rate of yiqiRunchangTongfu decoction was $100 \%$ and the therapeutic effect was better.

\subsection{Huanglong Decoction combined with acupuncture}

Fu Lingzhi et al. [34] believed that most constipation patients were qi deficiency and bowel dryness type, and the main treatment was to nourish qi, nourish blood, moisten intestines and relieve constipation. 60 cases were 
treated and observed, and the observation group was treated with Huanglong Decoction combined with acupuncture therapy. The basic ingredients of Huanglong soup are mirabilite, rhubarb, Angelica, Aurantii trifoliata, ginseng, Magnolia officinalis, prepared glycyrrhiza. This recipe is based on dachengqi decoction, which has the effects of replenishing qi and nourishing blood, regulating qi stagnation, moistening intestines and relieving constipation. The results showed that the total effective rate of the observation group was $91.7 \%$, which was much higher than that of the conventional oral polydiethyl alcohol treatment in the control group. It could quickly relieve or eliminate the symptoms of constipation and achieved ideal curative effect, and the recurrence rate was only 5.5\% after one month. Compared with western medicine, the prognosis of the combined treatment of Traditional Chinese medicine was better.

\subsection{Adding atractylodesatractylodes decoction}

Chen Lin and Zhang Yong [35] treated a total of 80 cases of qi-deficiency constipation with modified Baizhu Decoction to supplement deficiency and transport spleen according to gaoCaida's dialectical thought of "spleen deficiency does not carry”. Specific prescriptions for rhizomaatractylodis, rehmannia sativus, radish seed, hemp, paeony. Sheng Atractylodes is a jun medicine, invigorating the spleen and moistening the dryness, promoting the transport function of the spleen. This square tonifying qi Yin, through invigorating the spleen, promoting transport, lifting and lowering turbidity, so as to moisten the intestine and relieve constipation. The control group was treated with oral lactulose. The treatment group achieved good results, the total effective rate was $90 \%$, much higher than the control group.

In conclusion, there are many treatment methods for constipation, but there is still no unified treatment standard. Traditional Chinese medicine has obvious advantages over western medicine in the treatment of qi-deficiency constipation, with fewer toxic and side effects and good long-term effects. This paper summarizes the application of nourishing qi and moistening intestines in the treatment of chronic constipation by combing the understanding of traditional Chinese and western medicine on chronic constipation and the clinical application of buzhongYiqi decoction.

\section{References}

[1] Qin, G. Z. (2018). Traditional Chinese Medicine Anoenterology [M]. Beijing: Science Press, 2018: 115-126. (in Chinese)

[2] Wang Jiyao. (2011). Internal Medicine (2nd Ed.) [M]. Beijing: People’s Medical Publishing House, 2011: 479-481.

[3] Li Zengjin, et al. (2000). Prevalence of constipation in elderly patients in Beijing [J]. Chin J gerontology, 2000, 20(1): 1-2.

[4] Ye Fei, et al. (2010). Chronic constipation: an epidemiological study [J]. Chinese Journal of Clinical Health, 2010, 13(6): 665-666.

[5] Pu Yongping, Cao Jixun, et al. (2021). Treatment of female constipation from liver [J]. China Journal of Traditional Chinese Medicine Information, 2021, 28(2): 1-3.

[6] Chu Hui-ping, et al. (2013). Analysis of epidemiological characteristics and influencing factors of constipation in college students in Wuhan [J]. Gastroenterology, 2013, 18(8): 482-486.

[7] Fang Xiucai, et al. (2001). An epidemiological survey of defecation habits in non-patient adult population in Beijing [J]. Chin J med, 2001, 81(21): 1287-1290.

[8] Cui, Y. H. (2014). Discussion on the effect of environmental change on constipation in secondary school girls [J]. Health Vocational Education, 2014, 15(8): 127-128.

[9] Yu Prin, et al. (2001). A preliminary analysis of the epidemiological characteristics of constipation in the elderly [J]. Chinese Journal of Gerontology, 2001, 20(2): 132-134.

[10] Liu Yuchun. (2002). Treatment and prevention of opiate induced constipation [J]. Journal of Foreign Medical Nursing, 2002, 21(8): 392-393.

[11] Dong Jiarong, et al. (2012). Journal of Shandong University of Traditional Chinese Medicine, 2012, 36(3): $229-231$.

[12] Chen Hongfeng. (2016). Surgery of Traditional Chinese Medicine (4th edition) [M]. Beijing: China Traditional Chinese Medicine Press, 2016: 265-269.

[13] He Yufang. (2016). J clin Med, 2016, 3(16): 3327-3330. (in Chinese)

[14] Lu Ruimin. Clinical observation of Jiawei ShengyangYiwei Decoction (Shuimi pills) in treatment of senile habitual constipation [J]. China Science and Technology of Traditional Chinese Medicine, 2021, 28(1): 154-156.

[15] Chen Xuan, et al. (2020). Clinical efficacy of maren pill combined with lactulose in promoting functional constipation in the 
elderly [J]. Chinese Journal of Medical Research, 2020, 18(34): 134-136.

[16] Ning Yuyin, et al. Effect of WenyangYiqi ointment on improving senile functional constipation [J]. Journal of Changchun University of Traditional Chinese Medicine, 2021, 37(3).

[17] Wang Haifang, et al. (2020). Effect of new acupuncture patch combined with rhubarb patch on functional constipation in elderly patients [J]. Chin J Med Review, 2020, 17(31): 142-145.

[18] Huang Cuichang, et al. (2020). Nursing effect of auricular point pressing bean combined with hand acupoint massage on senile patients with qi-deficiency constipation [J]. General Nursing, 2020, 18(35): 4930-4932.

[19] Liu Li, et al. Safety and efficacy evaluation of acupuncture in the treatment of chronic functional constipation [J]. Liaoning Journal of Traditional Chinese Medicine.

[20] Lin Linyan, et al. (2020). Clinical observation on the treatment of qi deficiency constipation after stroke with mountain fire acupuncture [J]. Chinese Journal of External Treatment, 2020, 29(4): 57-59.

[21] Chang Shu Bao, et al. (2020). General situation of clinical research on treating constipation from deficiency in traditional Chinese medicine [J]. Hebei Journal of Traditional Chinese Medicine, 2020, 35(5): 57-61.

[22] Wang Jiarui, et al. (2020). Clinical efficacy of BuzhongYiqi Decoction in treating senile functional constipation with Qi deficiency: a meta-analysis [J]. Modern Medicine and Health, 2020, 36(1): 25-28.

[23] Feng Xingzhong, et al. (2014). Journal of Beijing University of Traditional Chinese Medicine, 2014, 37(1): 5-14.

[24] He Yanhua, et al. (2004). Journal of Traditional Chinese Medicine, 2004, 22(03): 9-10. (in Chinese)

[25] Tang Ruizhu, et al. (2020). Discussion on prevention and treatment of constipation based on invigorating spleen and invigorating qi [J]. Liaoning journal of traditional Chinese medicine, 2020, 47(8): 68-71.

[26] Xie, J. Y., Deng, X. M. Discussion on the cubic essence of BuzhongYiqi Decoction with "The theory of Yin-Yang rising and falling” [J]. Journal of Liaoning Traditional Chinese Medicine, 2019, 46(1): 54-56. (in Chinese with English abstract)

[27] Yuan Wenbei, Li Zhongzhuo, Liu Ycan. (2020). Clinical observation of buzhongyiqi decoction in the treatment of functional constipation [J]. Shanxi Traditional Chinese Medicine, 2020, 36(12): 17-19.

[28] Xiao Dinghong, Li Liang, Wang Qingqi. (2017). Chinese Journal of Basic Medicine of Traditional Chinese Medicine, 2017, 23(6): 862-864.

[29] Yao Yibo, et al. (2020). Clinical observation on the treatment of severe mixed constipation with Qi-Yin deficiency [J]. Journal of Shanghai University of Traditional Chinese Medicine, 2020, 34(6): 19-29.

[30] Wang, Y., Wang, Y., Wang, Y., et al. (2017). Effect of buzhongYiqi Decoction on chronic constipation in elderly patients [J]. Chinese Community Physicians, 2017, 33(33): 93-94.

[31] Liang Pei-ling. (2019). Clinical study on the treatment of chronic functional constipation with traditional Chinese medicine [J]. Guangming Traditional Chinese Medicine, 2019, 34(7): 2112-2114.

[32] Song Liying, et al. (2019). Effect of yiqirunchang prescription on senile functional constipation with qi deficiency [J]. Chinese Community Physicians, 2019, 35(1): 96-97.

[33] Hong Yanghua, et al. (2020). Effect of YiqiRunchangTongfu Decoction on qi deficiency syndrome of colon slow transit constipation [J]. Chinese Journal of Medical Research, 2020, 18(1): 1-3.

[34] Fu Lingzhi, et al. (2018). Clinical effect of acupuncture combined with Huanglong Decoction on 60 cases of Senile Qi Deficiency and intestinal dryness constipation [J]. World Journal of Integrated Chinese and Western Medicine, 2018, 13(7): 965-968.

[35] Chen Lin, et al. (2020). Effect of modified baizhu decoction on 80 cases of functional constipation [J]. Zhejiang Journal of Traditional Chinese Medicine, 2020, 55(8): 578-579. 